\title{
Theoretical studies of a hydrogen abstraction tool for nanotechnology
}

\author{
Charles B Musgravet, Jason K Perry†, Ralph C Merkleł and \\ William A Goddard III†§ \\ tMaterials and Molecular Simulations Center, Beckman Institute, California \\ Institute of Technology, Pasadena, CA91125, USA \\ $\ddagger$ Xerox Palo Alto Research Center, 3333 Coyote Hill Road, Palo Alto, CA 94304, \\ USA
}

Received 8 January 1992, accepted for publication 22 April 1992

\begin{abstract}
Processes that use mechanical positioning of reactive species to control chemical reactions by either providing activation energy or selecting between alternative reaction pathways will allow us to construct a wide range of complex molecular structures. An example of such a process is the abstraction of hydrogen from diamond surfaces by a radical species attached to a mechanical positioning device for synthesis of atomically precise diamond-like structures. In the design of a nanoscale, site-specific hydrogen abstraction tool, we suggest the use of an alkynyl radical tip. Using ab initio quantum-chemistry techniques including electron correlation we model the abstraction of hydrogen from dihydrogen, methane, acetylene, benzene and isobutane by the acetylene radical. Of these systerns, isobutane serves as a good model of the diamond (1111) surface. By conservative estimates, the abstraction barrier is small (less than $7.7 \mathrm{kcal} \mathrm{mol}^{-1}$ ) in all cases except for acetylene and zero in the case of isobutane. Thermal vibrations at room temperature should be sufficient to supply the small activation energy. Several methods of creating the radical in a controlled vacuum setting should be feasible. Thermal, mechanical, optical and chemical energy sources could all be used either to activate a precursor, which could be used once and thrown away, or alternatively to remove the hydrogen from the tip, thus refreshing the abstraction tool for a second use. We show how nanofabrication processes can be accurately and inexpensively designed in a computational framework.
\end{abstract}

\section{Introduction}

Mechanical positioning of reactive species can be used to convert mechanical energy to chemical energy to select between alternative reactions, or to provide activation energy. Mechanosynthesis is the employment of these mechanochemical processes to synthesize molecular structures [1]. Atomically precise mechanosynthesis promises to let us manufacture complex systems of molecular machinery. Examples include: self-replicating assemblers [2], molecular-scale surgical systems [2], computers made with molecular logic elements [3] and macroscopic machines made of diamond-like materials. Construction of such systems will require the ability to manipulate structure precisely on an atomic level. The great specificity of the chemical reactions required to synthesize designs with specific atomic structures should be achievable with mechanochemical tools capable of positioning the reactive moieties with sub-ångström accuracy. Mechanochemistry allows alternative reaction transition states to be selected by manoeuvring the

§uthor to whom all correspondence should be addressed. reactive species into a position where the chosen reaction has the smallest barriers. Such positional control requires that the tool exert forces and torques on the reactive molecule to move it over the potential energy surface of interaction with the workpiece.

Applying positional control to reactions will require that the tool have certain properties to make synthesis reliable, feasible and practicable. The tool must (i) have the proper chemical properties, (ii) be relatively small to reduce steric interactions with the workpiece, (iii) be capable of remaining chemically and mechanically stable under thermal motions and strains induced during positioning, (iv) be bound to a system that can transfer forces and torques to the reactive portion of the tool, (v) be selective between alternative reactions and (vi) be easily made. Molecular tips attached to atomic force microscope (AFM) tips, scanning tunnelling microscope (STM) tips or molecular robotic arms have been suggested [4].

Because construction of atomically precise machinery might require about as many unit operations as there are atoms in the system, it is important that reactions be fast. To increase the speed of reactions with moderate barriers, forces can be exerted between the workpiece and 
reactive species to effectively increase the pressure on the system, reducing the barrier height. Moderate reductions in the barrier heights lead to substantial increases in the reaction rate because thermal vibrations have an exponential Boltzmann probability of overcoming the reaction barrier. Mechanochemistry not only reduces the barriers by converting mechanical energy to chemical energy, but also maximizes the effective reactive concentration by positioning the reactive moieties to best advantage. These speed-enhancing steps together with having many mechanochemical machines working simultaneously can compensate for the loss of parallelism of solution-based reactions. Mechanochemical synthesis also increases the range of synthetic steps that can be used to build novel structures by the use of applied torques; a moiety attached to both the tool and the workpiece can be twisted, for example, to break $\pi$-bonds [1].

Nanomachines made of complex specific arrangements of diamond-like material offer several advantages. First, diamond is light and stiff. Macroscopic machines could be made stronger and simultaneously much lighter, making such activities as air and space travel substantially more practicable. Moving parts of such machines would be lighter and therefore faster. Furthermore, hydrocarbons are abundant, making raw materials readily available and inexpensive. Stiffness is not only a desirable property of finished machines, but it is also useful during construction since the material surrounding the reactive site on the workpiece must be stiff. This allows it to withstand the compressive forces that might be needed to reduce reaction barriers, to withstand the tensile forces during moiety abstraction and to withstand torques applied to break $\pi$-bonds.

Building machines of diamond will include manoeuvring hydrocarbons into reactive sites, torsion of structures, insertions into bonds and preparation of reactive sites by removing unwanted moieties to create radical sites. Abstraction of hydrogen is likely to be the most repeated step and common to building a wide range of molecular structures, including diamond-like structures. Highly reactive species are commonly thought to play a crucial role in the chemical vapour deposition (CVD) synthesis of diamond [5-7]. The abstraction of hydrogen via any of several radicals is one of the central mechanisms involved in the growth of diamond. It is not unreasonable, therefore, to expect that the atomically precise synthesis of diamond-like materials will utilize site-specific hydrogen abstraction via a radical as one of the main steps. Drexler has proposed using a molecular tip made of an ethynyl radical [1]

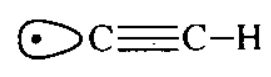

bound to a mechanical base on the tool (figure 1).

While many radicals exist, the desire for a simple, general, positionally accurate and sterically undemanding hydrogen abstraction tool can be used to narrow the search to a structure that (i) has a very high affinity for hydrogen, (ii) is not encumbered by surrounding groups,

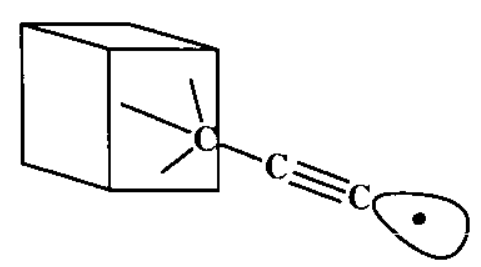

Figure 1. Acetylene radical hydrogen abstraction molecular tip attached to diamond-like material.

(iii) can be made part of an extended structure, which can be used as a 'handle' for positioning and can be attached to an STM or AFM tip, (iv) is mechanically and chemically stable during positioning, (v) is selective between alternative reactions such as abstraction of a neighbouring hydrogen or bonding to a nearby carbon atom and (vi) is easily made or regenerated. Perhaps the most natural structure in this regard is the ethynyl radical.

The $\mathrm{C}-\mathrm{H}$ bond in acetylene is one of the strongest bonds to hydrogen; thus, the ethynyl radical formed by removing this hydrogen is likely to have a higher affinity for hydrogen than almost any other chemical structure. Further, the ethynyl radical can easily be incorporated into structures that provide a high degree of steric exposure. A structure resembling the propynyl radical, but with the carbon furthest from the radical site embedded in an extended diamond-like structure (figure 1), provides both excellent steric exposure to the radical and a 'handle' for positioning the radical for the desired abstraction. Attachment of the tool to an STM or AFM tip may develop from technology designed for attachment of proteins to surfaces [4]. Drexler showed that the bending stiffness of an ethynyl-like tip attached to an adamantyl group is $\sim 6 \mathrm{~N} \mathrm{~m}^{-1}$ and can be increased to $\sim 65 \mathrm{~N} \mathrm{~m}^{-1}$ by building up a surrounding collar [1]. If the reaction requires application of mechanical force to supplement thermal energy, then bending stiffness may need to be increased. Stiffness also is desirable to achieve selectivity. STM and AFM positioning is stable to more than subångström accuracy. However, bending modes of the ethynyl tip will be active at moderate temperatures. If during positioning of the tip, the bending of the radical and displacement of the AFM or STM relative to the workpiece positions the reactive portion of the tip near a branched transition state, for example (one pathway leading to abstracting the neighbouring hydrogen), then selectivity is reduced. Drexler has shown that at worst at room temperature with a bending stiffness of $20 \mathrm{~N} \mathrm{~m}^{-1}$ and transition states separated by $1.2 \AA$ the unwanted reaction rate is less than $10^{-12}$ times the rate of the target reaction [1]. Transition states between neighbouring hydrogens on the (1 111 ) surface of diamond are separated by $2.5 \AA$, and transition states for other possible reactions in diamond-like structures also generally exceed $1.2 \AA$, making mechanochemical reactions highly selective.

The strong $\mathrm{C}-\mathrm{H}$ bond of alkynes (127$132 \mathrm{kcal} \mathrm{mol}^{-1}$ [8-12] should give large exothermicities and small barriers (rapid reactions) for alkynyl radical abstraction of hydrogen from weaker $\mathrm{sp}^{2}$ and $\mathrm{sp}^{3}$ hybridized $\mathrm{C}-\mathrm{H}$ bonds (see table 1). The large exothermicity 
Table 1. Experimental bond dissociation energies (kcal $\mathrm{mol}^{-1}$ ).

\begin{tabular}{lr}
\hline $\mathrm{R}-\mathrm{H}$ & \multicolumn{1}{c}{$D_{0}$} \\
\hline $\mathrm{H}-\mathrm{H}$ & $102.3[25]$ \\
$\mathrm{CH}_{3}-\mathrm{H}$ & $105.1[27]$ \\
$\left(\mathrm{CH}_{3}\right)_{3} \mathrm{C}-\mathrm{H}$ & $93.2[27]$ \\
$\mathrm{C}_{6} \mathrm{H}_{5}-\mathrm{H}$ & $110.9[27]$ \\
$\mathrm{HCC}-\mathrm{H}$ & $126.6[11], 131.3[9]$ \\
\hline
\end{tabular}

would also give a small reverse reaction rate constant. Compressive mechanical forces could be applied to supplement thermal energy in cases where the barriers are large; however, care must be taken so that the alkynyl radical tip does not bend away from the transition state. In the cases we study the barriers are such that this is not an issue.

Several methods of creating the radical should be feasible. The process of creating the radical should take place in an inert environment: vacuum, helium, or some other extremely non-reactive system would be appropriate. The activation energy required to create the abstraction tool could be provided from thermal, mechanical, optical, or chemical sources. There are two obvious approaches. In the first, a precursor compound is activated to create the abstraction tool. The tool is then used once and discarded. A second precursor would then be activated to abstract a second hydrogen. Thus, in a functioning system using this approach, a steady supply of the precursor would be required as well as a method for disposing of the 'used' abstraction tools.

In the second approach, the abstraction tool would be refreshed by the removal of the hydrogen after each use. Of course, the ethynyl radical was selected on the basis of its strong $\mathrm{C}-\mathrm{H}$ bond, so removal of the hydrogen might at first seem paradoxical. However, there are several methods of solving this problem. One would be first to weaken the $\mathrm{C}-\mathrm{H}$ bond, and then abstract the hydrogen from the abstraction tool using a weaker radical. Drexler [1] proposed that the $\mathrm{C}-\mathrm{H}$ bond could be weakened by positioning a weak radical near the carbon atom. A second weak radical could then abstract the hydrogen from the tip.

An alternative to the 'attack by two weak radicals' strategy would be photoexciting the acetylene to obtain the $\pi \pi^{*}$ state, which would rearrange to the structure<smiles>C=C</smiles>

with a weak $\mathrm{C}-\mathrm{H}$ bond and thereby allow removal of the hydrogen. Our primary concern is to analyse the energy barriers associated with hydrogen abstraction using alkynyl radicals to determine the feasibility of such a tool, rather than to analyse the methods of creating such a tool.

We model the chemically active site of the tool by the acetylene radical and determine the transition-state geometry and activation energy for transferring the hydrogen from several species: $\mathrm{H}_{2}, \mathrm{CH}_{4}, \mathrm{C}_{2} \mathrm{H}_{2}, \mathrm{C}_{6} \mathrm{H}_{6}$ and $\mathrm{CH}\left(\mathrm{CH}_{3}\right)_{3}$. The geometry of the various transition states can be used to position a working hydrogen abstraction tool for fast reaction and so as not to bend the tip. The barrier height itself can be used to calculate an abstraction rate at a given temperature and, thus, how long the abstraction tool must remain at the transition state until the probability that abstraction has occurred reaches a given value. Various levels of generalized valence-bond (GVB) and configuration-interaction (CI) $a b$ initio calculations are used. To calibrate the accuracy of these calculations, we consider the abstraction barriers and transition states for hydrogen transfer between methyl and methane and for hydrogen transfer between $\mathrm{H}$ and $\mathrm{H}_{2}$ as compared to other theoretical and experimental results [13-15].

\section{Results}

The barrier to the acetylene radical abstraction of hydrogen from isobutane ( $\mathrm{sp}^{3}$ carbon) is conservatively estimated to be less than $0.45 \mathrm{kcal} \mathrm{mol}^{-1}$ (figure 2). Reaction barriers calculated at various levels of correlation are shown in table 2. The barrier to abstraction of hydrogen by the acetylene radical from benzene $\left(s \mathrm{p}^{2}\right.$ carbon) is estimated to be less than $7.7 \mathrm{kcal} \mathrm{mol}^{-1}$. The Hartree-Fock times singles and doubles configuration interaction ( $\mathrm{HF} * \mathrm{SD} \mathrm{CI}$ ) consistently overestimates the generalized valence bond times singles and doubles configuration interaction (GVB $* \mathrm{SDCl}$ ) barriers, while the

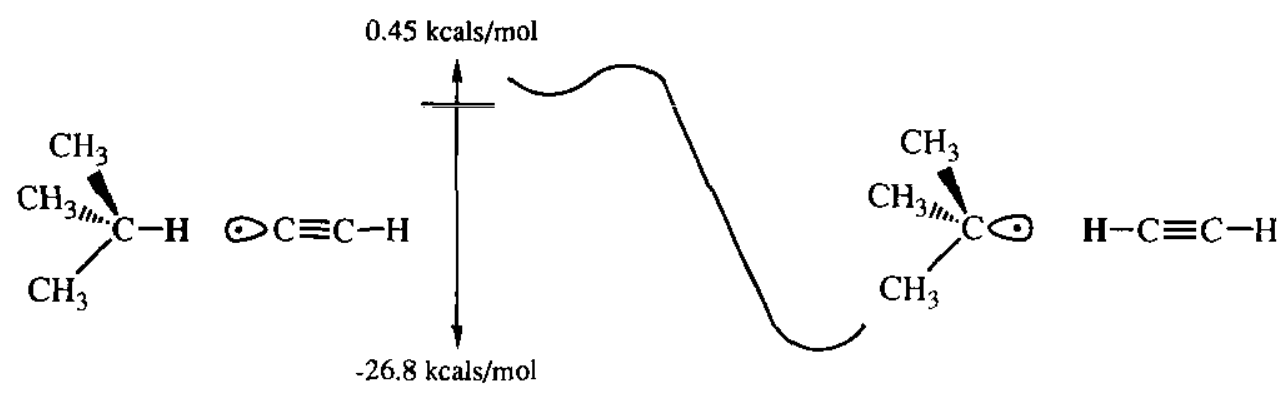

Figure 2. Schematic diagram showing the transition barrier and exothermicity of the acetylene radical abstraction of hydrogen from isobutane. 
Table 2. Barriers for the hydrogen abstraction reaction, $\mathrm{R}_{1}-\mathrm{H}+\mathrm{R}_{2} \rightarrow \mathrm{R}_{1}+\mathrm{H}-\mathrm{R}_{2}$, calculated at the cccl optimized geometry ( $\mathrm{kcal} \mathrm{mol}^{-1}$ ).

\begin{tabular}{lcccccc}
\hline $\mathrm{R}_{1}-\mathrm{H}-\mathrm{R}_{2}$ & $\mathrm{HF}$ & GVBCl-SCF & CCCI & DCCl & HF*SDCl & GVB*SDCl \\
\hline $\mathrm{H}-\mathrm{H}-\mathrm{H}^{\mathrm{a}}$ & 24.3 & 9.9 & 10.3 & 10.3 & $10.5(9.6)^{\mathrm{c}}$ & $10.3(10.3)$ \\
$\mathrm{CH}_{3}-\mathrm{H}-\mathrm{CH}_{3}$ & 34.9 & 27.8 & 29.8 & 17.5 & $22.5(19.5)$ & $20.4(18.8)$ \\
$\mathrm{H}-\mathrm{H}-\mathrm{CCH}$ & 11.6 & 8.0 & 5.4 & 0.8 & $4.5(3.2)$ & $3.3(2.7)$ \\
$\mathrm{CH}_{3}-\mathrm{H}-\mathrm{CCH}$ & 14.6 & 8.6 & 10.2 & $-2.9^{\mathrm{b}}$ & $4.2(2.0)$ & $2.2(1.3)$ \\
$\left(\mathrm{CH}_{3}\right)_{3} \mathrm{C}-\mathrm{H}-\mathrm{CCH}$ & 11.0 & 5.9 & 8.0 & -7.0 & $0.45(-2.78)$ & \\
$\mathrm{C}_{6} \mathrm{H}_{5}-\mathrm{H}-\mathrm{CCH}$ & 18.3 & 12.0 & 14.3 & -0.7 & $7.7(4.1)$ & \\
$\mathrm{HCC}-\mathrm{H}-\mathrm{CCH}$ & 30.0 & 22.9 & 24.1 & 11.6 & $17.0(13.7)$ & $14.6(12.9)$ \\
\hline
\end{tabular}

an the case of $\mathrm{H}-\mathrm{H}-\mathrm{H}$, the $\mathrm{CCCl}, \mathrm{DCCl}$ and $\mathrm{GVB} * \mathrm{SD} \mathrm{Cl}$ (three reference SDCl) are equivalent calculations.

${ }^{b} \mathrm{Negative}$ values indicate that the $\mathrm{CCCl}$ transition-state geometry is lower in energy than the reactants at this level of calculation. This suggests that no barrier exists on the potential energy surface.

"Numbers in parentheses are the barriers when the Davidson correction is included.

dissociation-consistent configuration interaction (DCCI) consistently underestimates the GVB*SD Cl barriers. The GVB $*$ SD CI barriers will be conservatively high due to a lack of a third diffuse $p$ function, zero-point corrections and lack of more correlation of the valence electrons. Transition states were optimized at the correlationconsistent configuration interaction (CCCI) level (table 3). Although $\mathrm{CCCl}$ does not accurately predict activation barriers, it does accurately describe the transition-state geometries. For the largest cases, the number of spin eigenfunctions in the configuration-interaction (CI) calculation grows beyond our computational capabilities (table 4). This makes abstraction from benzenc and isobutane at the GVB*SD CI level impracticable; however, the overestimated, yet small, barriers at the $\mathrm{HF} * \mathrm{SD}$ CI level show that the acetylene radical hydrogen abstraction is feasible, thermal vibrations at room temperature providing sufficient energy to overcome the barriers. Table 5 shows the exothermicities for the various abstractions. There is little difference in the accuracy of the methods in predicting the exothermicities because all the methods describe bound states rather well. Note that the exothermicities are for reactions where the product radical species are not allowed to relax. This describes abstraction from surfaces where relaxation is constrained. Exothermicities for gas-phase reactions will be higher. The transition state is poorly described by many semi-empirical methods and by ab initio methods with

Table 3. Transition-state geometries optimized at the cccl level. See text for constraints on these geometries.

\begin{tabular}{llll}
\hline $\mathrm{R}_{1}-\mathrm{H}-\mathrm{R}_{2}$ & $\begin{array}{l}R_{1 \mathrm{H}} \text { bond } \\
\text { length }(\hat{\mathrm{A}})\end{array}$ & $\begin{array}{l}R_{2 \mathrm{H}} \text { bond } \\
\text { length }(\hat{\mathrm{A}})\end{array}$ & $\begin{array}{l}\mathrm{H}-\mathrm{C}-\mathrm{C} \\
\text { angle }^{\mathrm{a}}(\mathrm{deg})\end{array}$ \\
\hline $\mathrm{H}-\mathrm{H}-\mathrm{H}$ & 0.94 & 0.94 & \\
$\mathrm{CH}-\mathrm{H}-\mathrm{CH}_{3}$ & 1.36 & 1.36 & 105.2 \\
$\mathrm{H}-\mathrm{H}-\mathrm{CCH}$ & 0.80 & 1.61 & 105.5 \\
$\mathrm{CH}_{3}-\mathrm{H}-\mathrm{CCH}$ & 1.22 & 1.48 & \\
$\left(\mathrm{CH}{ }_{3}\right)_{3} \mathrm{C}-\mathrm{H}-\mathrm{CCH}$ & 1.2 & 1.5 & \\
$\mathrm{C}_{6} \mathrm{H}_{5}-\mathrm{H}-\mathrm{CCH}$ & 1.24 & 1.42 & \\
$\mathrm{HCC}-\mathrm{H}-\mathrm{CCH}$ & 1.28 & 1.28 & \\
\hline
\end{tabular}

aThis is the angle between the methyl hydrogens and the $\mathrm{C}-\mathrm{H}-\mathrm{C}$ axis.

Table 4. Number of spin eigenfunctions in each of the $\mathrm{Cl}$ calculations. The numbers in parentheses are the number of spin eigenfunctions for the separated cases, $R_{1}-H+R_{2}$, where symmetry is broken.

\begin{tabular}{lcccc}
\hline $\mathrm{R}_{1}-\mathrm{H}-\mathrm{R}_{2}$ & $\mathrm{CCCl}$ & $\mathrm{DCCl}$ & $\mathrm{HF} * \mathrm{SDCl}$ & $\mathrm{GVB} * \mathrm{SDCl}$ \\
\hline $\mathrm{H}-\mathrm{H}-\mathrm{H}$ & $191(386)$ & $191(386)$ & $139(278)$ & $191(386)$ \\
$\mathrm{CH}-\mathrm{H}-\mathrm{CH}_{3}$ & $4914(9829)$ & $71666(143333)$ & $79428(158757)$ & $310778(621164)$ \\
$\mathrm{H}-\mathrm{H}-\mathrm{CCH}$ & 2160 & 18234 & 15189 & 53048 \\
$\mathrm{CH}_{3}-\mathrm{H}-\mathrm{CCH}$ & 9477 & 150557 & 184851 & 737651 \\
$\left(\mathrm{CH}_{3}\right)_{3} \mathrm{C}-\mathrm{H}-\mathrm{CCH}$ & 21676 & 695842 & 1675566 & \\
$\mathrm{C}_{6} \mathrm{H}_{5}-\mathrm{H}-\mathrm{CCH}$ & 17265 & 587343 & 1514151 & \\
$\mathrm{HCC}-\mathrm{H}-\mathrm{CCH}$ & $2697(5398)$ & $42201(84406)$ & $55211(110314)$ & $221805(443182)$ \\
\hline
\end{tabular}


Table 5. Calculated exothermicities $\left(\mathrm{kcal} \mathrm{mol}^{-1}\right)$.

\begin{tabular}{lllllll}
\hline $\mathrm{R}_{1}-\mathrm{H}-\mathrm{R}_{2}$ & $\mathrm{HF}$ & GVBCI-SCF & $\mathrm{CCCl}$ & $\mathrm{DCCl}$ & $\mathrm{HF} * \mathrm{SDCl}$ & GVB*SDCl \\
\hline $\mathrm{H}-\mathrm{H}-\mathrm{CCH}$ & 33.9 & 31.6 & 31.8 & 30.1 & $30.0(27.5)^{\mathrm{a}}$ & $29.9(28.1)$ \\
$\mathrm{CH}_{3}-\mathrm{H}-\mathrm{CCH}$ & 28.9 & 28.4 & 27.0 & 29.2 & $26.9(25.5)$ & $26.8(25.4)$ \\
$\left(\mathrm{CH}_{3}\right)_{3} \mathrm{C}-\mathrm{H}-\mathrm{CCH}$ & 28.5 & 27.9 & 24.2 & 27.4 & $26.8(25.9)$ & \\
$\mathrm{C}_{6} \mathrm{H}_{5}-\mathrm{H}-\mathrm{CCH}$ & 18.7 & 18.2 & 15.4 & 18.9 & $18.3(18.0)$ & \\
\hline
\end{tabular}

aNumbers in parentheses include the Davidson correction.

insufficient electron correlation and small basis sets, and large variation of the predicted barriers can be seen in table 2 .

\section{Calculational details}

Standard $a b$ initio quantum-chemistry methods are employed and results are given for several levels of calculation. The simplest wavefunction used is the wavefunction in which each molecular orbital is doubly occupied. This single-configuration (one-determinant) Hartree-Fock (HF) wavefunction is the lowest-energy antisymmetrized $n$-fold product of molecular orbitals and should give a qualitative picture of the hydrogen abstraction reactions studied. HF will tend to overestimate the abstraction barrier since the radical-hydrogen stretching frequency is too high due to the poor description by HF of the bond breaking process (a doubly occupied orbital of the molecule must become two singly occupied orbitals for the fragments). This problem is remedied by using a GVB (generalized valence-bond) wavefunction [16], which allows each bond to be described with two singly occupied, overlapping orbitals leading to a proper description of dissociation. When solved for self-consistently, this calculation is termed a generalized valence-bond configuration-interaction selfconsistent field (GVBCI-SCF) or equivalently a complete active space self-consistent field (CASSCF). Simply, all symmetry- and spin-allowed configurations of three active electrons in three orbitals are generated. These electrons are the radical of the reactant, the hydrogen and the radical of the product. All other orbitals (considered inactive) are doubly occupied as in HartreeFock. It is found that three configurations are all that is necessary to describe the transition state adequately. These are the dominant configuration (with the HartreeFock occupations of the orbitals) and the single and double excitations of the electrons in the doubly occupied $\mathrm{R}_{1}-\mathrm{H}-\mathbf{R}_{2}$ bonding orbital to the empty $\mathbf{R}_{1}-\mathbf{H}-\mathbf{R}_{2}$ antibonding orbital. The $\mathbf{R}_{1}-\mathbf{R}_{2}$ antibonding orbital (with a node at the hydrogen centre) is singly occupied in all three configurations. While this level of calculation includes the most important correlation, it will still tend to overestimate the abstraction barrier and, thus, will only serve as a zeroth-order wavefunction for large CI (configuration-interaction) expansions, which will account for additional dispersion.

Ideally, we would like to do a Cr calculation in which all single and double excitations of the valence electrons are made into the virtual orbitals with reference to the three most important configurations describing the abstraction. This type of multi-reference CI (called a $\mathrm{GVB} * \mathrm{SD} \mathrm{CI})$ has been well established in approximating results of complete $\mathrm{Cr}$ calculations [17]. However, this $\mathrm{CI}$ has not been carried out for the largest cases, abstraction of hydrogen from isobutane and benzene by the acetylene radical. Thus we have considered some smaller $\mathrm{CI}$ calcủations, which will do a good job in appioximating the barriers for the larger $\mathrm{CI}$. The first of these is the $\mathrm{CCCI}$ wavefunction $[18,19]$. It involves making all single and double excitations of the active electrons and all single excitations of the other valence electrons into the virtual space relative to the three GVB references, or simply GVB $*\left(S_{\text {active }}+S_{\text {valence }}\right)$. The second $\mathrm{CI}$, called a DCCI (dissociation-consistent configuration interaction), will add the double excitations, which are the product of a single excitation of an active electron and a single excitation of a valence electron, or $G \mathrm{~GB} *\left(\mathrm{SD}_{\text {active }}\right.$ $\left.+\mathrm{S}_{\text {active }} * \mathrm{~S}_{\text {valence }}+\mathrm{S}_{\mathrm{valence}}\right)$. The third CI does all single and double excitations of the valence electrons (active and inactive) relative to only one reference, a calculation called HF*SDCI (or equivalently one-reference SDCI). Table 4 shows the sizes of the CI expansions in terms of the number of spin eigenfunctions (SEF) for each of the systems studied. The $\mathrm{HF}_{*} \mathrm{SD} \mathrm{CI}$ already approaches the limits of our programs (about two million SEF) in the cases of isobutane and benzene, for which the GVB*SD CI is not possible. In all other cases, however, the GVB * SD CI are of small to medium size and will serve as benchmarks to calibrate the accuracy of the smaller $\mathrm{CI}$.

The standard basis sets of Dunning and Huzinaga are used $[20,21]$. Their double-zeta contraction of the $9 \mathrm{~s} 5 \mathrm{p}$ set is used on all carbons, with the addition of one set of $d$ polarization functions $\left(\zeta^{\mathrm{d}}=0.75\right)$. On the active carbons, diffuse $\mathrm{s}$ and $\mathrm{p}$ functions $\left(\zeta^{\mathrm{s}}=0.0474\right.$ and $\left.\zeta^{\mathrm{p}}=0.0365\right)$ are also added. For active hydrogens or hydrogens bound to active carbons (in the case of methane), the triple-zeta contraction of the $6 s$ set is used, supplemented with a $p$ polarization function $\left(\zeta^{\mathbf{p}}=0.60\right)$. For all other hydrogens, the double-zeta contraction of the $4 \mathrm{~s}$ set is used, scaled by a factor of 1.2 .

The basic geometries of the various systems studied are illustrated schematically in figure 3 . The geometries will be optimized at the CCCI level. The orbital optimization at the GVBCI-SCF level is the most time-consuming step, so this $\mathrm{CI}$ will be a simple correction to that wavefunction. It would be impracticable to do the 
System

$$
\begin{aligned}
& \mathrm{H}-\mathrm{H}-\mathrm{H} \\
& \mathrm{H}-\mathrm{H}-\mathrm{C} \equiv \mathrm{C}-\mathrm{H} \\
& \mathrm{H}-\mathrm{C} \equiv \mathrm{C}-\mathrm{H}-\mathrm{C} \equiv \mathrm{C}-\mathrm{H}
\end{aligned}
$$<smiles>C</smiles><smiles>C#C</smiles><smiles>[AsH2]</smiles><smiles>C#CC(C)C</smiles>

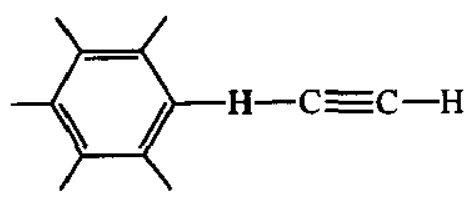

Figure 3. Geometries for the transition states.

geometry optimization at a higher-level CI. It would also be impracticable to do a full geometry optimization, so certain constraints are assumed. Namely, only the parameters relevant to the description of the hydrogen abstraction $\left(R_{1 \mathrm{H}}\right.$ and $R_{2 \mathrm{H}}$ or combinations thereof) will be optimized. In the case of abstraction from methane (by the methyl radical or by the acetylene radical), the $\mathrm{H}-\mathrm{C}-\mathrm{H}^{\text {abs }}$ bond angle is also optimized since this angle changes from $109.5^{\circ}$ for methane to $90^{\circ}$ for the methyl radical. For isobutane, we would expect a small relaxation from a tetrahedral $\mathrm{C}-\mathrm{C}-\mathrm{H}^{\mathrm{abs}}$ bond angle to something more planar at the transition state and in the radical species. But Page and Brenner [22], in their work on abstraction of hydrogen from isobutane by atomic hydrogen, found that full relaxation of the $t$-butyl species reduced the abstraction barrier by only $1.7 \mathrm{kcal} \mathrm{mol}^{-1}$ at the GVBCI-SCF level. However, since the goal of this work is to show the feasibility of using an alkynyl radical tip as a hydrogen abstraction tool, a conservative overestimate of the abstraction barriers is acceptable. So the $\mathrm{C}-\mathrm{C}-\mathrm{H}^{\text {abs }}$ bond angle is fixed to $109.5^{\circ}$ in these calculations. All other radicals are expected to show little or no relaxation and are fixed to the experimental values of their hydrogen bound counterparts.

All calculations are run with the GVB [23] and MOLECULE-SWEDEN (an electronic structure program system written by Almlöf et al [24]) suites of programs on the Caltech group's Alliant FX/80 and FPS 500.

\section{Discussion}

\section{1. $\mathrm{H}-\mathrm{H}-\mathrm{H}$}

A great deal of theoretical work has been done on this system [15] and, owing to its simplicity, it provides a good test of our hydrogen basis set and, to a lesser extent, our methods. While the calculated equilibrium bond distance in $\mathrm{H}_{2}$ compares favourably with experiment ( $0.74 \AA$ vs. $0.74144 \AA[25]$ ), the calculated dissociation energy for $\mathrm{H}_{2}\left(D_{\mathrm{e}}\right)$ is $105.4 \mathrm{kcal} \mathrm{mol}^{-1}$ comparcd with the experimental $108.6 \mathrm{kcal} \mathrm{mol}^{-1}$ [25]. This discrepancy of $3 \mathrm{kcal} \mathrm{mol}^{-1}$ is chiefiy due to the lack of a second $\mathrm{p}$ polarization function. In contrast, the transition state is well described by the GVB*SDCl (in this case only, the $\mathrm{CCCl}$, DCCI and $\mathrm{GVB} * \mathrm{SDCI}$ are equivalent since there are no valence electrons in addition to the three active electrons). The optimized geometry has a $\mathrm{H}-\mathrm{H}$ distance of $0.94 \AA$ (compared to Liu's value of $0.930 \AA$ [15]) and a barrier of $10.3 \mathrm{kcal} \mathrm{mol}^{-1}$ (compared to Bauschlicher's value of $9.56 \mathrm{kcal} \mathrm{mol}^{-1}$ using a large atomic natural orbital (ANO) basis set [15]). The geometries for all the systems studied are listed in table 3 and the abstraction barriers are listed in table 2 . An error of less than $1 \mathrm{kcal}$ in the barrier is adequate for the calculations at hand, particularly since the barrier is overestimated. It should be noted, however, that the Hartree-Fock barrier is well off the mark at $24.3 \mathrm{kcal} \mathrm{mol}^{-1}$ and that the apparently good result at the GVBCI-SCF level $\left(9.9 \mathrm{kcal} \mathrm{mol}^{-1}\right)$ is primarily due to the weak $\mathrm{H}-\mathrm{H}$ bond strength $\left(87.5 \mathrm{kcalmol}^{-1}\right)$ at this level. The $\mathrm{HF}^{*} \mathrm{SD} \mathrm{Cl}$ number including the Davidson correction is in fortuitous agreement with the reference barrier height of $9.56 \mathrm{kcal} \mathrm{mol}^{-1}$. This is rather symptomatic of the Davidson correction, which can often overestimate the contributions from additional correlation.

\section{2. $\mathrm{CH}_{3}-\mathrm{H}-\mathrm{CH}_{3}$}

The methyl-methane hydrogen transfer reaction is perhaps more representative as a test case of the systems in which we are most interested. There has been less theoretical work done on this system [14] but a reliable experimental number for the abstraction barrier of $14.2 \mathrm{kcal} \mathrm{mol}^{-1}$ [13] gives a good benchmark for us to work with. Theoretical investigations into this reaction have not been successful in obtaining quantitative accuracy in the barrier height. The best calculations overestimate the barrier by $5-6 \mathrm{kcal} \mathrm{mol}^{-1}$. Part of this is due to some assumptions made in the calculations, namely the neglect of zero-point corrections, the BornOppenheimer approximation and temperature effects. However, the sum of these effects should only lower the theoretical activation energy by $1-2 \mathrm{kcal} \mathrm{mol}^{-1}$ (see Sana et al [14]). Our results agree well with previous 
theoretical work. The optimized transition-state geometry is virtually identical to the full gradient optimized structure of Wünsch et al [14], which was done at the Hartree-Fock level. The calculated barrier is $20.4 \mathrm{kcal} \mathrm{mol}^{-1}$, higher than experiment by $6.2 \mathrm{kcal} \mathrm{mol}^{-1}$. Test calculations with a large ANO basis set [26] only lowered the barrier to $19.2 \mathrm{kcal} \mathrm{mol}^{-1}$, indicating that the discrepancy between theory and experiment is probably a correlation problem rather than a basis set problem. The importance of ionic terms such as $\left(\mathrm{CH}_{3}-\mathrm{H}^{+}-{ }^{-} \mathrm{CH}_{3}\right)$ are probably underestimated in the CI calculations owing to biases against anionic states and would require additional correlation of the nonactive valence electrons. This could be a formidable task even for such a small system and would not be possible with our current code for the larger cases we wish to study. However, again, since these factors all tend to lead to an overestimate of the barrier height, the results for abstraction of hydrogen by the acetylene radical can be considered a conservative upper limit to the actual barrier height. These calculations on the methylmethane system also offer a comparison of the smaller $\mathrm{CI}$ to the GVB*SD standard. We find the CCCI result $\left(29.8 \mathrm{kcal} \mathrm{mol}^{-1}\right)$ to be comparable to the GVBCI-SCF number $\left(27.8 \mathrm{kcal} \mathrm{mol}^{-1}\right)$, being slightly higher due to the stronger $\mathrm{C}-\mathrm{H}$ bond at the $\mathrm{CCCI}$ level $\left(113.2 \mathrm{kcal} \mathrm{mol}^{-1}\right.$ vs. $97.6 \mathrm{kcal} \mathrm{mol}^{-1}$ at the GVBCI-SCF level and $107.2 \mathrm{kcal} \mathrm{mol}^{-1}$ at the GVB*SDCI level). This indicates that correlation of the non-active electrons is important in obtaining quantitative accuracy for the hydrogen abstraction barriers. The DCcl, which includes only limited correlation of the inactive electrons, underestimates the GVB*SD CI barrier by $2.9 \mathrm{kcal} \mathrm{mol}^{-1}$. Alternatively, the $\mathrm{HF} * \mathrm{SD} \mathrm{CI}$, which sacrifices some of the active electron correlation, overestimates this barrier by $2.1 \mathrm{kcal} \mathrm{mol}^{-1}$. The combination of these two $\mathrm{CI}$ calculations should offer an upper and lower limit to the GVB*SD CI for those large cases where that $\mathrm{CI}$ is not feasible.

\section{3. $\mathrm{H}-\mathrm{H}-\mathrm{CCH}, \mathrm{CH}_{3}-\mathrm{H}-\mathrm{CCH}, \mathrm{C}_{6} \mathrm{H}_{5}-\mathrm{H}-\mathrm{CCH}$ and $\mathrm{HCC}-\mathrm{H}-\mathrm{CCH}$}

Results of calculations on these systems underscore the results of methyl-methane. In particular, Hartree-Fock greatly overestimates the activation energies, GVBCI-SCF and $\mathrm{CCCI}$ offer some improvement but still overestimate these barriers, and DCCI and HF *SD CI bracket the results of the GVB * SD Cl. In the case of abstraction from methane and benzene, we found that the geometries were most conveniently optimized by using the coordinate system $R_{1 \mathrm{H}}+R_{2 \mathrm{H}}$ and $R_{1 \mathrm{H}}-R_{2 \mathrm{H}}$. In the case of abstraction from $\mathrm{H}_{2}$, it was easier to optimize the transition state in terms of the coordinates $R_{1 \mathrm{H}}$ and $R_{2 \mathrm{H}}$. This was probably due to the fact that the barrier was small at the CCCI level and that it occurred quite early, with only an $8 \%$ increase in the $\mathrm{H}-\mathrm{H}$ bond length. To a large degree the barrier height and the position of the barrier are determined by the exothermicity of the reaction. Other properties, such as the polarizability of the bonds, play a role as well. The largest barrier (and latest transition state) was for $a b$ straction from acetylene, with an activation energy of $14.6 \mathrm{kcal} \mathrm{mol}^{-1}$. This is as expected, since this particular reaction is thermoneutral. The calculated exothermicities of the other reactions are listed in table 5 (see also table 1 , for the experimental bond dissociation energies). $\mathrm{H}_{2}$ and $\mathrm{CH}_{4}$ are the most exothermic and have the smallest barriers. These barriers may be considered negligible as the calculations on $\mathrm{H}+\mathrm{H}_{2}$ and methyl-methane showed the numbers to be overestimated. Abstraction from benzene is less exothermic and shows a small but non-negligible barrier. In addition the phenyl- $\mathrm{H}$ bond is stretched $14 \%$ at the transition state in comparison to an $11 \%$ stretch of the methyl- $\mathbf{H}$ bond. All of these results correlate with the exothermicities of the reactions.

\section{4. $\left(\mathrm{CH}_{3}\right)_{3} \mathrm{C}-\mathrm{H}-\mathrm{CCH}$}

Finding the transition state for abstraction from isobutane proved to be quite difficult. The potential energy surface has many of the same features as that of $\mathrm{H}-\mathrm{H}-\mathrm{CCH}$, in particular, a small, early barrier that leads to non-quadratic behaviour in the region of the saddle point. Owing to the computational costs of these calculations, it was necessary to sacrifice some accuracy in order to find the transition state. In the end, the values of $R_{1 \mathrm{H}}=1.2 \AA$ and $R_{2 \mathrm{H}}=1.5 \AA$ are in agreement with results for abstraction from methane and benzene by the acetylene radical. The large CL calculations strongly indicate that there is no barrier for abstraction of hydrogen, the more conservative number giving a barrier of only $0.45 \mathrm{kcal} \mathrm{mol}^{-1}$. This again correlates with the large exothermicity of this reaction, which is calculated from snap bond energies. If one considers that the $t$-butyl group should relax somewhat at the CCCI transition state and, thus, lower the energy of the barrier still, the argument for the absence of a barrier becomes even more persuasive. Since this system is a good model for the hydrogenated diamond ( 1111 ) surface $\mathrm{C}-\mathrm{H}$ bond, we conclude that no barrier exists to abstraction of hydrogen from this surface by acetylene. Barriers on other surfaces of diamond are probably non-existent or negligibly small.

\subsection{Abstraction from acetylene}

Now that it has been established that an alkynyl-tipped hydrogen abstraction tool would be able to abstract hydrogen from diamond surfaces with little or no thermodynamic hindrances, it would be desirable to find a method for removing the hydrogen from the tip. A simple alternative, but less elegant strategy is to make a new tip for each abstraction and dispose of the tool after use. What makes the acetylene good at abstracting hydrogen is the strength of its $\mathrm{C}-\mathrm{H}$ bond. However, this bond is quite weak in the ${ }^{3} \mathrm{~B}_{\mathrm{u}}$ excited state (see table 6). We calculate a bond strength of $41.7 \mathrm{kcal} \mathrm{mol}^{-1}$ doing a GVBCI-SCF in which all 10 valence electrons are active in 10 orbitals, followed by a multi-reference times singles and doubles configuration interaction ( $\mathrm{MR} * \mathrm{SDCI}$ ) in 
Table 6. Calculated relevant energetics of ground-state acetylene and the excited-state triplet ( $\left.\mathrm{ccal} \mathrm{mol}^{-1}\right)$.

\begin{tabular}{lrcc}
\hline & HF & GVBCI-SCF & \multicolumn{1}{c}{ MR*SD Cl } \\
\hline$T_{\mathrm{e}}\left({ }^{3} \mathrm{~B}_{\mathrm{u}} \leftarrow{ }^{1} \Sigma_{\mathrm{g}}^{+}\right)$ & 72.3 & 92.0 & $90.2(87.6)$ \\
$D_{\mathrm{e}}\left({ }^{\prime} \Sigma_{\mathrm{g}}^{+} \mathrm{HCC}-\mathrm{H}\right)$ & 116.5 & 122.3 & $131.9(132.0)$ \\
$D_{\mathrm{e}}\left({ }^{3} \mathrm{~B}_{\mathrm{u}} \mathrm{HCC}-\mathrm{H}\right)$ & 44.2 & 30.3 & $41.7(44.4)$ \\
\hline
\end{tabular}

which all configurations in the GVBCI with coefficients $>0.05$ are chosen as references. In the case of the triplet excited state, there are four references and, in the case of dissociated $\mathrm{H}+\mathrm{CCH}$, there are six references. The geometry for the excited state is optimized at the $\mathrm{MR} * \mathrm{SDCI}$ level. The molecule is not linear in this state, having a $\mathrm{C}-\mathrm{C}-\mathrm{H}$ bond angle of $132.0^{\circ}$. The $\mathrm{C}-\mathrm{C}$ bond length also increases to $1.38 \AA$ from $1.20 \AA$ for the ground state, reflecting the double bond character of this bond. The weakening of the $\mathrm{C}-\mathrm{C}$ bond in the excited state leads directly to the weakening of the $\mathrm{C}-\mathrm{H}$ bonds, as the triple bond character can be restored upon dissociation of one of the $\mathrm{C}-\mathrm{H}$ bonds. The weakening of the $\mathrm{C}-\mathrm{H}$ bond leaves the acetylene prone to abstraction, making it easy to remove the hydrogen and refresh the tip. So photoexcitation of the alkynyl tip from its ground state to the ${ }^{1} B_{u}$ excited state, followed by relaxation to the triplet would facilitate the breaking of the tip-hydrogen bond. Drexler also made an alternative proposal for removing the hydrogen from the tip [1].

\section{Conclusion}

We model the abstraction of hydrogen from $\mathrm{H}_{2}, \mathrm{CH}_{4}$, $\mathrm{C}_{2} \mathrm{H}_{2}, \mathrm{C}_{6} \mathrm{H}_{6}$ and $\mathrm{CH}\left(\mathrm{CH}_{3}\right)_{3}$ by the acetylene radical using accurate $\mathrm{Cl}$ ab initio quantum-chemistry techniques. From our results, conservative estimates show that the reaction barriers for abstraction from $\mathrm{sp}^{3}$ hybridized carbons are negligible, or zero for the case of isobutane. The barriers are small for $\mathrm{sp}^{2}$ hybridized carbons and slightly larger for sp hybridized carbons. Therefore, abstraction tools based on ethynyl radical molecular tips should reliably and rapidly abstract hydrogen from most carbon structures at moderate temperatures. We also find that the hydrogen bond to the $\pi-\pi^{*}$ excited enthynyl tip is relatively weak, and therefore can be broken to refresh the tip. We have also shown how nanofabrication processes can be accurately and inexpensively designed in a computational framework.

\section{Acknowledgments}

We gratefully acknowledge the useful discussions with $\mathrm{K}$ Eric Drexler, Siddharth Dasgupta and Erik P Bierwagen. CBM was supported by a Department of Defense National Defense Science and Engineering Grant (NDSEG) fellowship and JKP was partially supported by a BP America fellowship. This research was partially supported by the Xerox Corporation. The facilities of the
Materials and Molecular Simulations Center were supported by grants from NSF (NSF-CHE-9100284) and ONR/NRL, and by grants from Allied-Signal, BP America, Asahi Chemical, Asahi Glass, General Electric, General Motors, Chevron and Xerox.

\section{References}

[1] Drexler K E 1992 Nanosystems: Molecular Machinery, Manufacturing, and Computation (New York: Wiley)

[2] Drexler K E 1986 Engines of Creation (New York: Anchor Press/Doubleday)

[3] Drexler K E 1988 Molecular Electronic Devices (Amsterdam: North-Holland)

[4] Drexler K E 1991 J. Vac. Sci. Technol. B 91394

[5] Celii F G, Pehrsson P E, Wang H and Butler J E 1988 Appl. Phys. Lett. 522043

[6] Harris S J, Weiner A M and Perry T A $1988 \mathrm{Appl}$. Phys. Lett. 531605

Harris S J and Weiner A M 1990 J. Appl. Phys. 67 6520

[7] Goodwin D G and Gavillet G G 1989 J. Appl. Phys. 68 6393

[8] Wodtke A M and Lee Y T 1985 J. Phys. Chem. 89 4744

[9] Ervin K M, Gronert S, Barlow S E, Gilles M K, Harrison A G, Bierbaum V M, Depuy C H, Lineberger W C and Ellison G B $1990 \mathrm{~J}$. Am. Chem. Soc. 1125750

[10] Segal J, Wen Y, Lavi R, Singer R and Wittig C J 1991 J. Phys. Chem. 958078

[11] Green P G, Kinsey J L and Field R W $1989 \mathrm{~J}$. Chem. Phys. 915160

[12] Segal J, Lavi R, Wen Y and Wittig C J 1989 J. Phys. Chem. 937287

[13] Tedder J M 1982 Angew. Chem. Int. Ed. Engl. 21401

[14] Wünsch E, Lluch J M, Oliva A and Bertrán J $1987 \mathrm{~J}$. Chem. Soc. Perkin Trans. II 211

Sana M, Leroy G and Villaveces J L 1984 Theor. Chim. Acta 65109

Rayez-Meaume M T, Dannenberg J J and Whitten J L 1978 J. Am. Chem. Soc. 100747

[15] Liu B J 1973 Chem. Phys. 581925

Bauschlicher C W, Langhoff S R and Partridge H 1990 Chem. Phys. Lett. 170345 and references therein

Siegbahn P and Liu B J 1978 Chem. Phys. 682457

[16] Bobrowicz F W and Goddard W A 1977 Methods of Electronic Structure Theory ed H F Schaefer (New York: Plenum) p 79

[17] Bauschlicher C W and Langhoff S R 1987 Chem. Phys. Lett. 13567

Taylor P R and Partridge H 1987 J. Phys. Chem. 91 6148

For an excellent review see Bauschlicher C W, Langhoff S R and Taylor P R 1990 Adv. Chem. Phys. 77103

[18] Carter E A and Goddard W A 1988 J. Chem. Phys. 88 1752

[19] Carter E A and Goddard W A 1988 J. Chem. Phys. 88 3132

[20] Dunning T H 1970 J. Chem. Phys. 532823

[21] Huzinaga S J 1965 Chem. Phys. 421293

[22] Page M and Brenner D W $1991 \mathrm{~J}$. Am. Chem. Soc. 113 3270

[23] Goddard III W A et al, Caltech, unpublished

[24] Almlöf J, Bauschlicher C W, Blomberg M R A, Chong D P, Heiberg A, Langhoff S R, Malmqvist P-A, Rendell A P, Roos B O, Siegbahn P E M and Taylor P R MOLECULE-SWEDEN (program) unpublished 
[25] Huber K P and Herzberg G 1979 Molecular Structure IV: Constants of Diatomic Molecules (New York: Van Nostrand Reinhold)
[26] Almlöf J and Taylor P R 1987 J. Chem. Phys. 864070

[27] McMillen D F and Golden D M 1982 Annu. Rev. Phys. Chem. 33493 